\title{
Cortisol Supplement Combined with Psychotherapy and Citalopram Improves Depression Outcomes in Patients with Hypocortisolism after Traumatic Brain Injury
}

\author{
Lanlan Luo ${ }^{1,2}$, Yan Chai ${ }^{2}$, Rongcai Jiang ${ }^{2,3}$, Xin Chen ${ }^{2,3}$,Tao Yan ${ }^{1,2, *}$ \\ ${ }^{1}$ Neurology of Tianjin Medical University General Hospital, Tianjin, China \\ ${ }^{2}$ Tianjin Neurological Institute, Tianjin, China \\ ${ }^{3}$ Neurosurgery of Tianjin Medical University General Hospital, Tianjin, China
}

[Received April 15, 2015; Revised May 6, 2015; Accepted May 7, 2015]

\begin{abstract}
Depression is one of the most prevalent psychiatric disorders in people with Traumatic brain injury (TBI). Depression after TBI is closely related with social and psychological factors and hypothalamicpituitary -adrenal (HPA) axis dysfunction. However, there is a lack of evidence regarding effective treatment approaches for depression. A total of 68 patients with depression following closed TBI were recruited. Glasgow Coma Scale score (GCS) was employed to demonstrate the severity of neurological deficits and Glasgow Outcome Scale (GOS) was employed to measure functional outcome after TBI. The severity of depression was quantified using the Beck Depression Inventory-II (BDI-II) in line with DSM-IV. Citalopram and Prednisone were administered to subjects with normal cortisol levels or hypocortisolism separately, based on psychotherapeutic interventions. We investigated the relationship between degree of depression of TBI patients and the severity and progression of TBI with the therapeutic effects of Citalopram in combination with psychotherapeutic and Prednisone in depressed patients. There was no relationship between the severity of depression and the severity and progression of TBI. The basic treatment of psychotherapeutic interventions could partially relieve depressive symptoms. Combination of psychotherapeutic support and Citalopram significantly improved depressive symptoms in patients with normal cortisol levels, but not in hypocortisolic patients. Combination of Prednisone administration with psychotherapeutic treatment and Citalopram significantly improved depression outcome in hypocortisolic patients after TBI. Hypocortisolism after TBI may regulate depression. Combination of Prednisone with psychotherapeutic treatment and Citalopram may provide better therapeutic effects in depression patients with hypocortisolism after TBI.
\end{abstract}

Key words: traumatic brain injury, depression, citalopram, cortisol supplement

Traumatic brain injury (TBI) affects approximately 10 million people annually worldwide[1]. TBI is a major cause of death and disability and may result in thinking, memory, movement, sensation deficits, or psychiatric sequel[2]. Approximately $40 \%$ of TBI survivors suffer from psychological and psychosocial disturbances, including personality change, apathy, insomnia, aggression, anxiety, mania, psychosis and major depression $[3,4]$.

Depression appears to be one of the most prevalent and disabling psychiatric disorders in people with TBI[5]. The prevalence of depression after TBI ranges from $6 \%$ to $77 \%$ according to different assessment methods [6-9]. Symptoms of depression after TBI include depressed mood, lack of energy, suicidal attempts, irritability, loss of interest, frustration, fatigue, sleep disturbance, and poor concentration $[10,11]$. These effects last long-term and may impose a substantial impact on the quality of life and are a global burden [12]. Depression following TBI is associated with impaired sociability during the first year

*Correspondence should be addressed to: Dr. Tao Yan, Neurology of Tianjin Medical University General Hospital, 
post injury [13], as well as, cognitive impairment, increasingly severe post-concussion symptoms (such as uncoordinated gait, generalized weakness, headache, blurred vision, dizziness as well as learning and memory deficits) and lower health related quality of life [14]. However, the relationship between depressive symptoms and severity of TBI or recovery after TBI are uncertain[15]. Mixed results of a positive link, no relationship, or an inverse link between TBI severity and depressive symptoms have been published by previous studies $[6,9,13,16]$. More data may provide stronger evidence to understand the relationship between TBI and depression in its various forms and severity. In addition, understanding the relationship between TBI severity and depressive symptoms may provide a full spectrum of possible outcomes after TBI, and help understand whether TBI is a risk factor for depression.

Many biological and psychosocial factors contribute to depression after TBI. A fundamental question is which treatment approaches will be the most effective. Psychosocial factors are important for depression. In addition, hypopituitarism is a neuroendocrine sequela among TBI survivors and nearly half the patients with hypopituitarism suffered from mild to moderate depression [17]. Previous studies have found that cortisol disturbances are related with the development of depression. Hypercortisolemia is more prevalent in depression than hypocortisolemia, which is also commonly seen after TBI [18]. There are large numbers of cortisol receptors in the hippocampus, frontal cortex, and other areas. Any events affecting these areas could have significant effects on mood [19, 20]. Excessive, chronic, exposure to high levels of glucocorticoids (GC) such as Cushings Syndrome (CS), has multiple adverse effects on brain biology in animals and humans. In CS, chronic, stress-level concentrations of cortisol lead to depressed mood in over $60 \%$ of patients[21], as well as cognitive dysfunction, especially in memory [22]. Hypercortisolemic depression may be characterized by more "typical" depressive symptoms, such as hyperarousal, anxiety, insomnia, and loss of appetite [23]. Previous studies have found that normalization of cortisol levels improves the patient's mood and reduces anxiety symptoms[24], as well as increases memory and hippocampal volume[25, 26].

Low plasma cortisol concentrations were extremely common in TBI patients. Hypocortisolemia is related with subsequent mortality and prediction of chronic hypopituitarism [27]. In addition, hypocortisolemia may lead to a characterized depression with "atypical" symptoms of hypoarousal, hypersomnia, hyperphagia, lethargy, pain, fatigue, and relative apathy [28]. Owing to the hypothesized side effects of glucocorticoid therapy, insufficient treatment strategy and hypocortisolemia as a simple marker of high mortality, replacement of cortisol is still an uncertain treatment in TBI patients with low plasma cortisol $[27,29]$. Whether combination treatment with Prednisone (a corticosteroid replacement drug), psychotherapeutic interventions and anti-depression drugs improves depression after TBI is not clear.

In this study, we investigated the relationship between degree of depression in TBI patients, and the severity and progression of TBI. We also investigated the therapeutic effects of combination of Prednisone with psychotherapeutic interventions and anti-depression drugs in TBI patients with depression.

\section{MATERIALS AND METHODS}

\section{Subjects}

A total of 68 subjects identified with clinically diagnosed depression and self-reports of depression (clinically significant cases or depression scale scores) were recruited in their first follow-up visit after being discharged from the neurosurgery department of Tianjin Medical University General Hospital and Tianjin Huanhu Hospital from January 2010 to February 2014. The study was approved by the Institutional Review Board of Tianjin Medical University.

\section{The inclusion criteria for this study include:}

1) Age at injury greater than 16 years old;

2) Admission to hospital after TBI;

3) No history of suicide attempts, psychiatric hospitalization and were not under the active care of a psychiatrist;

4) Consent was obtained from either the patient or legally authorized representative.

\section{The exclusion criteria include:}

1) Pre-existing neurological or psychiatric disorders, including prior history of TBI, in an attempt to eliminate any other cause other than the current TBI for alterations in imaging, consciousness or cognition;

2) Presence of focal lesions (including contusions, extraaxial hematoma, and/or intraparenchymal hemorrhages) on cranial CT which could provide an alternative explanation for the decline in cognition or consciousness;

3) An open-head injury.

A total of 68 patients with depression following closed traumatic brain injury were recruited for this study. The 68 subjects consisted of 45 males and 23 females aged between 18 to 70 years old. 


\section{Severity of TBI}

The Glasgow Coma Scale score (GCS) was employed to determine the severity of neurological deficits after TBI when the patients were admitted to the hospital. The GCS is a quick, reproducible scoring system used during the initial examination to estimate TBI severity, it consists of three components (eye opening, verbal response, and the best motor response) for assessing depth of impaired consciousness and coma [30-36]. It was administered by the neurologist or the emergency room personnel in their initial evaluation, and had a score range from 3 to 15 . Patients with lower scores represent severe deficit, while higher initial scores tend to predict better recovery.

In this study, the severity of head injury is initially defined by the GCS. 1) 3-8 GCS scores are considered severe TBI; 2) 9-12 GCS scores considered moderate TBI and 3) 13-15 GCS scores considered mild TBI[36]. All those determined to have mild TBI met the mild TBI (MTBI) criteria of the American Congress of Rehabilitation as defined by GCS. Mild TBI is defined as a traumatically induced physiological disruption of brain manifested by at least one of the following: (a) any period of loss of consciousness; (b) any loss of memory for events immediately before or after the accident; (c) any alteration in mental state at the time of the accident (e.g., feeling dazed, disoriented, or confused); and (d) focal neurological deficit(s) that may or may not be transient. Meanwhile, the severity of injury does not exceed the following: loss of consciousness for approximately 30 minutes or less; after 30 minutes, an initial GCS of 13 to 15; and posttraumatic amnesia not greater than 24 hours [37].

\section{Neurological Outcome Measurement}

The Glasgow Outcome Scale (GOS) was employed to measure functional recovery after TBI before discharge from hospital for all TBI patients [38]. A five-point scale was used: 1) Death; 2) Persistent vegetative state; 3) Severe disability; 4) Moderate disability; and 5) Good recovery. It was developed by Jennett and Bond, and has since become firmly established as the primary outcome measure used in the majority of Phase III trials in TBI [39]. Since the first and second grades of GOS are death and persistent vegetative state, the depression condition of these patients could not be measured. Therefore, to test co-relationship of functional recovery after TBI with the degree of depression, the other three GOS grades were employed for correlation analysis.

\section{Diagnosis and Assessment of Depression}

All patients were evaluated by a psychiatrist using the structured clinical interview for Diagnostic and Statistical Manual of Mental Disorders (DSM) -IV [40]. Symptoms such as depressed mood, lack of energy, suicidal attempts, loss of interest, irritability, fatigue, sleep disturbance, frustration and poor concentration, were observed and recorded in these patients with TBI. Patients were diagnosed to have 'Depression' if they met the criteria for Mood disorder secondary to General Medical Condition, Major Depressive-like episode or other Depressivesymptoms. This method has been shown to be reliable for the categorical construct for DSM-IV diagnoses. These symptoms were recorded separately and showed as the percent frequency of occurrence.

Severity of depression was quantified using the Beck Depression Inventory-II (BDI-II) in line with the current depression criteria of DSM-IV [41]. It has shown high internal consistency, good test-retest reliability, and good construct and concurrent validity with other common measures of depression in clinical and nonclinical samples[42]. BDI-II is a widely used 21-item questionnaire for severity of depression symptoms. Each item can be scored from 0-3 and the total score can vary from 0 to 63 . BDI-II scores between $0-13$ are considered as no depression; 14-19 BDI-II scores as mild depression; 20-28 BDI-II scores as moderate depression; and 29-63 BDI-II scores as severe depression [43].

The measures are accomplished by a multidisciplinary team (endocrinologists, neurosurgeons, neurologists and psychiatrists) of Tianjin Medical University General Hospital. The follow-up time varies from 0.5 to 3 years.

\section{Treatment for the subjects}

Based on the strong and evolving evidence from previous studies $[44,45]$, the treatment included anti-depression pharmacological interventions, psychotherapeutic interventions and Prednisone. 1) Anti-depression pharmacological interventions: Citalopram (Cipramil, 20mg/tablet, 12 tablet, Lundbeck Denmark), which is an orally administered selective serotonin reuptake inhibitor (SSRI). Citalopram was administrated at a dose of $20 \mathrm{mg}$, Qd for 3 weeks or increased to $30 \mathrm{mg}$, Qd to ameliorate depression symptoms. 2) Psychotherapeutic interventions According to depression symptoms, several different types of psychotherapeutic interventions were employed in this study namely: interpersonal therapy, behavioral activation, cognitive-behavioral therapy, problem solving therapy, social skills training, psychodynamic therapy, and supportive counseling [46]. These psychotherapeutic interventions were performed by psychologists, rehabilitation doctors and social workers via face-to-face conversation. Patients had 1-hour of weekly psychotherapy sessions for six weeks after first being diagnosed with depression. 3) Prednisone treatment: 
Prednisone $(5 \mathrm{mg} /$ tablet, 100 tablets, Tianjin Lisheng, China), a corticosteroid replacement drug, was administrated (5 $\mathrm{mg} \mathrm{AM}$ and $2.5 \mathrm{mg} \mathrm{PM}$ ).

The therapeutic efficacy is described as complete remission, responder/improvement and invalid. Complete remission is defined as complete or near-complete absence of the symptom criteria of depression. On the BDI-II Scale, asymptomatic was operationalized as a score of 13 and below. Responder/improvement was defined as a $50 \%$ or greater reduction in scores on the clinician rating scale of BDI-II. An invalid was defined as less than a $50 \%$ reduction in scores on the clinician rating scale of BDI-II.

\section{Statistical analysis}

Descriptive statistics are presented as mean values \pm SE. Statistical analysis was done using non-parametric Kruskal-Wallis test. Correlations between various parameters in patients were analyzed using Pearson correlation coefficient. Analyses were performed using SPSS software (SPSS for Windows, release 11.5). $p$ values $<0.05$ were considered to be statistically significant.

\section{RESULTS}

\section{Symptoms of depression after TBI}

In this study, depression was observed from 3 months to 3 years after TBI. The symptoms of depression included insomnia 54 (54/68, 79.41\%), depressed mood 47 (47/68, $69.12 \%)$, poor concentration $33(33 / 68,48.53 \%)$ and fatigue $32(32 / 68,47.06 \%)$. In addition, thought retardation, headache, loss of interest, frustration, and memory loss were shown in some of these subjects. The severity of depression for the 68 subjects was divided into three groups: 1) mild depression: 22 (22/68, 32.35\%) patients; 2) moderate depression: 37 (37/68, 54.41\%) patients; and 3) severe depression: 9 (9/68, 13.24\%) patients.

There is no relationship between the severity of depression and severity of TBI

To test the effect of TBI severity on depression, a series of Pearson correlation coefficients were performed. We found no significant relationship between the severity of depression and severity of TBI (GCS grade) ( $\mathrm{r}=0.128$, $\mathrm{p}>0.05$, Fig. 1). Furthermore, there was no significant difference in the severity of depression among the three GCS grades of TBI patients $(H=0.129, \mathrm{p}>0.05$; Fig. 1$)$.

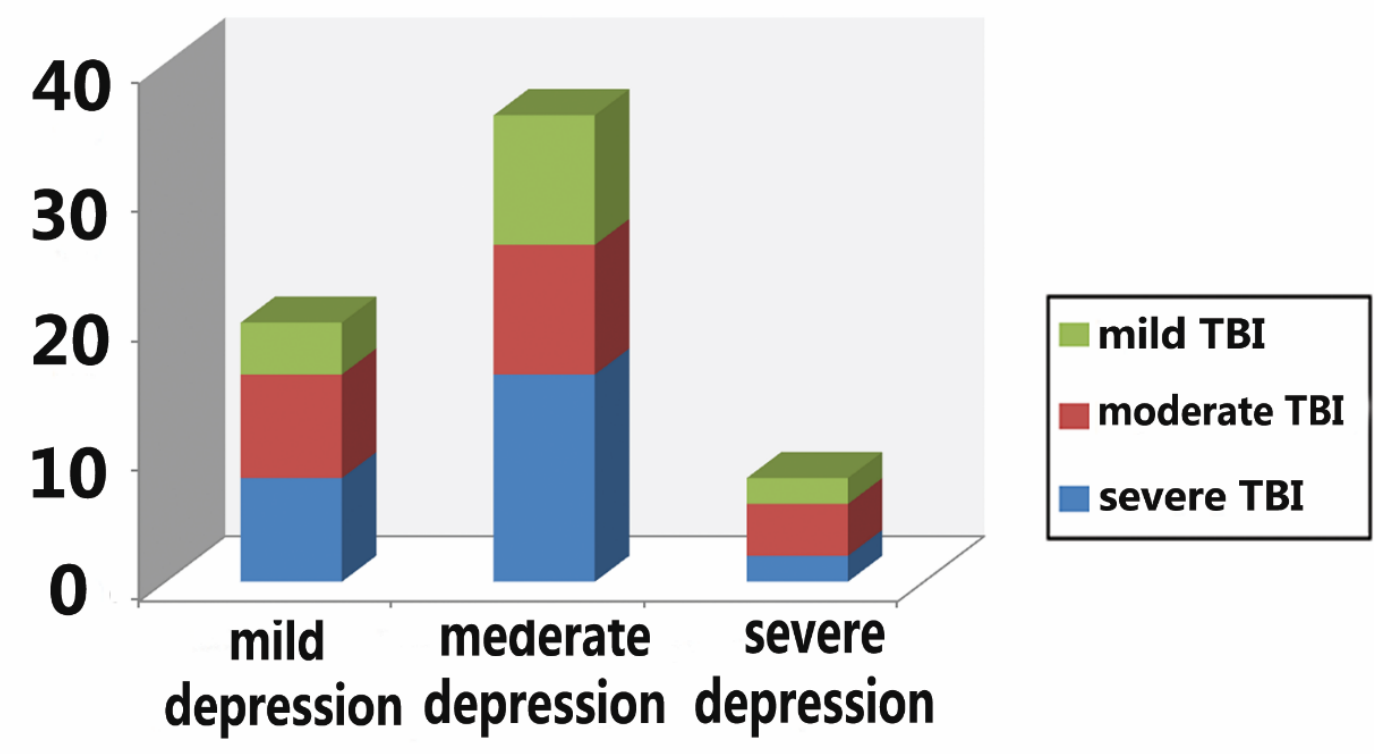

Figure 1. The distribution of severity of depression and severity of TBI. There is no significant relationship between the severity of depression and severity of TBI (GCS grade) $(r=0.128, p>0.05)$ and there is no significant difference for the severity of depression among the three GCS grades (grades 3-5) of TBI patients $(H=0.129, \mathrm{p}>0.05)$. 


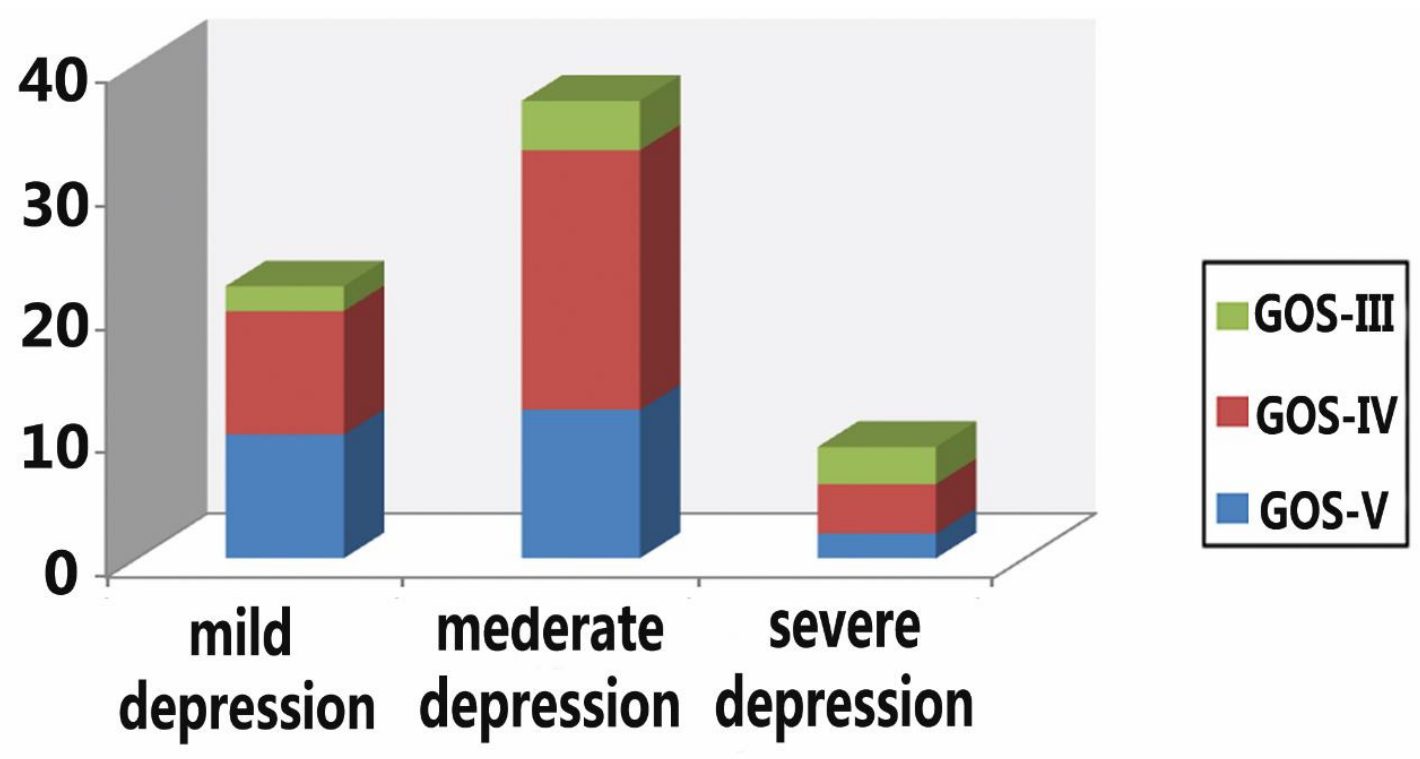

Figure 2. The distribution of severity of depression and TBI recovery score. There is no significant relationship between the severity of depression and GOS grade of TBI outcome $(r=0.206, p>0.05)$ and there is no significant difference for the severity of depression among three GOS grades (grades 3-5) groups $(\mathrm{H}=4.898, \mathrm{p}>0.05)$.

There is no relationship between the severity of depression and progression of TBI

To test the effect of progression and functional outcome after TBI on depression, a series of Pearson correlation coefficients between GOS score and depression degree were performed. There was no significant relationship between the severity of depression and GOS grade of TBI outcome ( $\mathrm{r}=0.206, p>0.05$, Fig. 2$)$. In addition, there was no significant difference for the severity of depression among three GOS grades (grades 3-5) groups $(\mathrm{H}=4.898$, $p>0.05$; Fig. 2).

\section{Therapeutic effects of combination treatment depression symptoms in patients after TBI}

1) Psychotherapeutic support:

All patients (68 subjects) were provided psychotherapeutic support. It was observed that 8 subjects $(8 / 68,11.76 \%)$ recovered from depression after TBI with psychotherapeutic support without pharmacological interventions, as well as $16(16 / 68,23.53 \%)$ subjects which showed a response and $44(44 / 68,64.70 \%)$ subjects showed no response to psychotherapeutic support. These 60 subjects were treated with a combination of Citalopram and psychotherapeutic support for patients with normal cortisol levels (28/60) and a combination of Citalopram, psychotherapeutic support and Prednisone for patients with hypocortisolism (32/60) (Fig. 3).

2) Citalopram combination with psychotherapeutic support:

28 patients with normal cortisol levels were treated with citalopram based on psychotherapy assessment. Among these 28 subjects, 18 (18/28, 64.29\%) subjects developed complete remission; $9(9 / 28,32.14 \%)$ subjects showed a decrease of more than $50 \%$ of the original BDIII scale; and $1(1 / 28,3.57 \%)$ subject did not show any improvement after combination treatment (Fig. 3).

3) Combination of Citalopram, Psychotherapeutic support and Prednisone treatment:

Previous studies have found that hormone deficiency regulates the development of depression after TBI $[17,18]$. We found that 32 subjects $(32 / 68,47.06 \%)$ had hypocortisolism (data not shown). Therefore, Prednisone and citalopram treatments were employed in these 32 patients who showed no beneficial effects from psychotherapy support. The data showed that $20(20 / 32$, $62.5 \%)$ subjects had complete remission; 10 (10/32, $31.25 \%$ ) subjects showed a decrease of more than $50 \%$ of the original BDI-II scale; and $2(2 / 32,6.25 \%)$ subjects did not show any improvement after combination treatment (Fig. 3). 


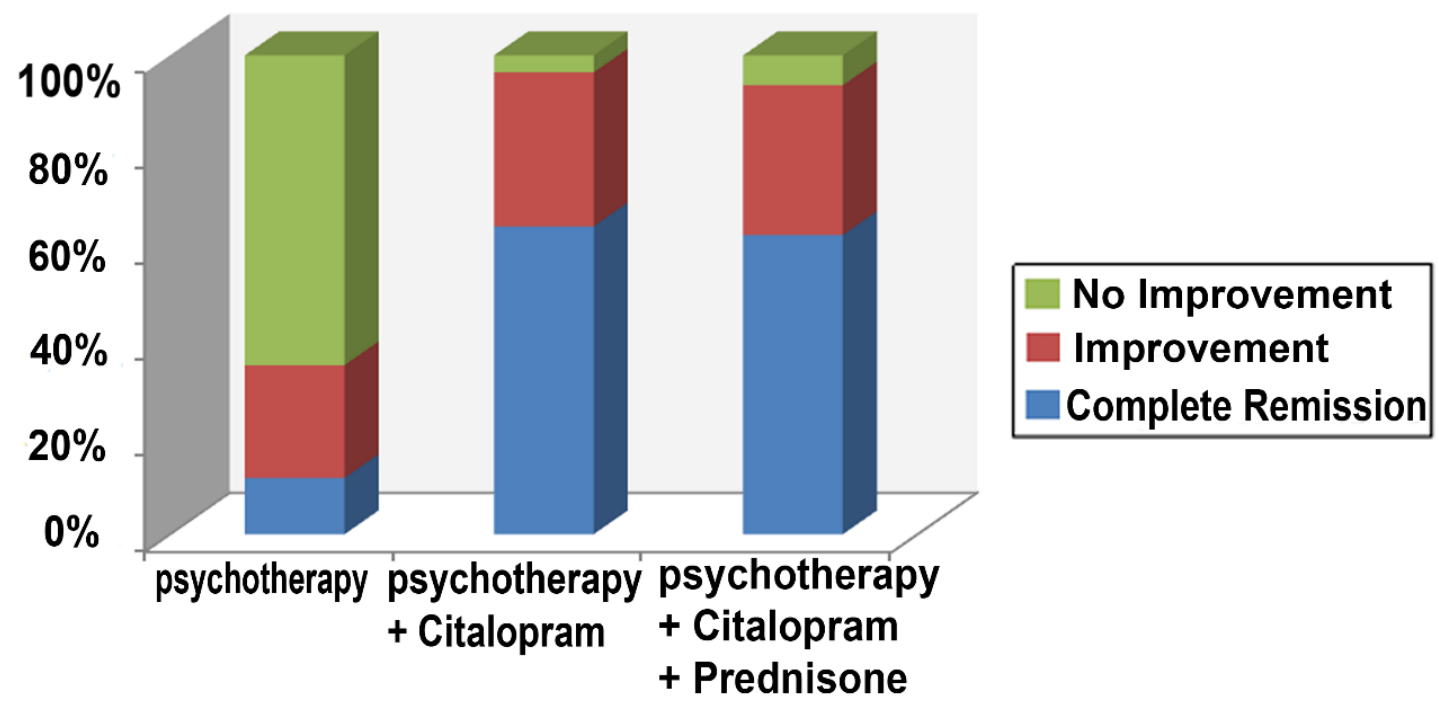

Figure 3. The distribution of therapy of depression and therapeutic effects in TBI patients. 8 subjects recovered from depression after TBI with psychotherapeutic support without pharmacological interventions. Citalopram was administrated to 28 patients with normal cortisol levels but showed no beneficial effects of psychotherapy support; combination treatment of prednisone and citalopram was employed in 32 patients with hypocortisolism.

\section{DISCUSSION}

Depression is one of the most frequently reported psychological problems following TBI. However, the clinical symptoms are easily misidentified as they include: depression, fatigue, poor concentration, irritability and a decline in overall cognitive functioning. Depression has the potential to impact on recovery and quality of life. Therefore, understanding the symptoms of depression after TBI may provide important information for depression treatment after TBI. Previous studies have found that there is about $52 \%$ prevalence rate for probable major depressive disorder (MDD) within the first year after injury[47]. In this study, depression symptoms were observed 3 months to 3 years after TBI. Among the depression patients, depression symptoms included insomnia (79.41\%), depressed $\operatorname{mood}(69.12 \%)$, poor concentration (48.53\%) and fatigue $(47.06 \%)$. In addition, few patients experienced mental retardation, headache, loss of interest, frustration, and memory loss. Insomnia, depressed mood and difficulty in concentration are the main clinical features of depression after TBI. In this study we also found that the degree of depression after TBI is not related with TBI severity and prognosis. Survivors of TBI with depression exhibit deterioration in social situations and performance of normal activities of daily living [48]. Therefore, treatment of TBI depression is important to improve day to day life after TBI.
Depression after TBI is closely related with social and psychological factors. In addition, the hypothalamicpituitary-adrenal (HPA) axis dysfunction may play a central role in stress and depression regulation. Depression being a stress-induced disorder is intimately related with stress. The HPA axis abnormalities are involved in depression [49]. Patients with hypocortisolism tend to present more distress symptoms, maladaptive coping styles, and schizotypal personality traits [50]. TBI patients induce HPA axis dysfunction in the acute phase of severe TBI patients. There are significantly lower glucocorticoid levels within 2.4 days after TBI and glucocorticoid levels decrease around 4 days after TBI [8]. Hypothalamic-pituitary dysfunction may be an important reason for the rehabilitation of TBIinduced depression. Therefore, supportive psychotherapeutic interventions combined with cortisol supplements to modulate HPA axis dysfunction may be an effective means to improve a patient's depression after TBI. In this study, in addition to psychotherapy and supportive antidepressant drug treatment, cortisol replacement was administrated to depression patients with hypocortisolemia following TBI. This combination treatment significantly improved the symptoms of depression. Therefore, apart from psychotherapy and supporting antidepressant drug treatment, modulating HPA axis is important for treatment of depression in patients following TBI. Wider adoption of the consensus 
guidelines on the detection and monitoring of endocrine abnormalities post-TBI in the clinic may provide a guideline for treatment of depression and diminish the severity of depression symptoms and ultimately improve quality of life [51].

Cortisol administration showed efficacy as a treatment for depressed patients with hypocortisolemia following TBI. However, hypocortisolemia is only one of the endocrine abnormalities which results in depressive symptoms in post-TBI patients. Further studies investigating the role of other hormones of the HPA axis on the development, prediction and progression of depression after TBI and the mechanisms via which Prednisone administration improves depression symptoms in patients with hypocortisolism are required in the future.

There are a few limitations to the present study. Although in this study, we have demonstrated that the combination of Citalopram with psychotherapeutic support improves depression outcome in TBI patients, whether citalopram treatment alone can improve depression outcome after TBI has not been investigated and future studies are warranted.

\section{Conclusion:}

Depression is one of the most frequently reported psychological problems following TBI. The degree of depression after TBI is not related with TBI severity and prognosis. Combination treatment of depression with psychotherapy, supportive antidepressant drugs and cortisol replacement may enhance treatment benefits.

\section{Acknowledgements}

This work was supported by grants from the National Natural Science Foundation of China (No.81300993, $81370029,81271359)$

\section{References}

[1] Hyder AA, Wunderlich CA, Puvanachandra P, Gururaj G, Kobusingye OC (2007). The impact of traumatic brain injuries: a global perspective. NeuroRehabilitation, 22: 341-353

[2] Langlois JA, Rutland-Brown W, Wald MM (2006). The epidemiology and impact of traumatic brain injury: a brief overview. J Head Trauma Rehabil, 21: 375-378

[3] Anstey KJ, Butterworth P, Jorm AF, Christensen H, Rodgers B, Windsor TD (2004). A population survey found an association between self-reports of traumatic brain injury and increased psychiatric symptoms. Journal of clinical epidemiology, 57: 1202-1209

[4] Vaishnavi S, Rao V, Fann JR (2009). Neuropsychiatric problems after traumatic brain injury: unraveling the silent epidemic. Psychosomatics, 50: 198-205

[5] Hibbard MR, Uysal S, Kepler K, Bogdany J, Silver J (1998). Axis I psychopathology in individuals with traumatic brain injury. J Head Trauma Rehabil, 13: 24-39

[6] Kreutzer JS, Seel RT, Gourley E (2001). The prevalence and symptom rates of depression after traumatic brain injury: a comprehensive examination. Brain injury, 15: 563-576

[7] Fedoroff JP, Starkstein SE, Forrester AW, Geisler FH, Jorge RE, Arndt SV, et al. (1992). Depression in patients with acute traumatic brain injury. The American journal of psychiatry, 149: 918-923

[8] Bowen A, Neumann V, Conner M, Tennant A, Chamberlain MA (1998). Mood disorders following traumatic brain injury: identifying the extent of the problem and the people at risk. Brain injury, 12: 177-190

[9] Rosenthal M, Christensen BK, Ross TP (1998). Depression following traumatic brain injury. Archives of physical medicine and rehabilitation, 79: 90-103

[10] Jorge RE, Robinson RG, Starkstein SE, Arndt SV (1993). Depression and anxiety following traumatic brain injury. The Journal of neuropsychiatry and clinical neurosciences, 5: 369-374

[11] Cavallo MM, Kay T, Ezrachi O (1992). Problems and changes after traumatic brain injury: differing perceptions within and between families. Brain injury, 6: 327-335

[12] Feigin VL, Theadom A, Barker-Collo S, Starkey NJ, McPherson K, Kahan M, et al. (2013). Incidence of traumatic brain injury in New Zealand: a populationbased study. The Lancet. Neurology, 12: 53-64

[13] Jorge RE, Robinson RG, Arndt SV, Starkstein SE, Forrester AW, Geisler F (1993). Depression following traumatic brain injury: a 1 year longitudinal study. Journal of affective disorders, 27: 233-243

[14] Fann JR, Katon WJ, Uomoto JM, Esselman PC (1995). Psychiatric disorders and functional disability in outpatients with traumatic brain injuries. The American journal of psychiatry, 152: 1493-1499

[15] Hudak AM, Hynan LS, Harper CR, Diaz-Arrastia R (2012). Association of depressive symptoms with functional outcome after traumatic brain injury. J Head Trauma Rehabil, 27: 87-98

[16] Jorge RE, Robinson RG, Moser D, Tateno A, CrespoFacorro B, Arndt S (2004). Major depression following traumatic brain injury. Archives of general psychiatry, 61: 42-50

[17] Popovic V, Aimaretti G, Casanueva FF, Ghigo E (2005). Hypopituitarism following traumatic brain injury. Growth hormone \& IGF research : official journal of the Growth Hormone Research Society and the International IGF Research Society, 15: 177-184

[18] Popovic V, Pekic S, Pavlovic D, Maric N, Jasovic-Gasic M, Djurovic B, et al. (2004). Hypopituitarism as a consequence of traumatic brain injury (TBI) and its possible relation with cognitive disabilities and mental distress. Journal of endocrinological investigation, 27: 1048-1054

[19] Sapolsky RM (2000). Glucocorticoids and hippocampal atrophy in neuropsychiatric disorders. Archives of general 
psychiatry, 57: 925-935

[20] Sapolsky RM (2000). The possibility of neurotoxicity in the hippocampus in major depression: a primer on neuron death. Biological psychiatry, 48: 755-765

[21] Starkman MN, Schteingart DE, Schork MA (1981). Depressed mood and other psychiatric manifestations of Cushing's syndrome: relationship to hormone levels. Psychosomatic medicine, 43: 3-18

[22] Forget H, Lacroix A, Somma M, Cohen H (2000). Cognitive decline in patients with Cushing's syndrome. Journal of the International Neuropsychological Society : JINS, 6: 20-29

[23] Wong ML, Kling MA, Munson PJ, Listwak S, Licinio J, Prolo P, et al. (2000). Pronounced and sustained central hypernoradrenergic function in major depression with melancholic features: relation to hypercortisolism and corticotropin-releasing hormone. Proc. Natl. Acad. Sci. U. S. A., 97: 325-330

[24] Starkman MN, Schteingart DE, Schork MA (1986). Correlation of bedside cognitive and neuropsychological tests in patients with Cushing's syndrome. Psychosomatics, 27: 508-511

[25] Starkman MN, Giordani B, Gebarski SS, Berent S, Schork MA, Schteingart DE (1999). Decrease in cortisol reverses human hippocampal atrophy following treatment of Cushing's disease. Biological psychiatry, 46: 1595-1602

[26] Starkman MN, Giordani B, Gebarski SS, Schteingart DE (2003). Improvement in learning associated with increase in hippocampal formation volume. Biological psychiatry, 53: $233-238$

[27] Hannon MJ, Crowley RK, Behan LA, O'Sullivan EP, O'Brien MM, Sherlock M, et al. (2013). Acute glucocorticoid deficiency and diabetes insipidus are common after acute traumatic brain injury and predict mortality. The Journal of clinical endocrinology and metabolism, 98: 3229-3237

[28] Anisman H, Ravindran AV, Griffiths J, Merali Z (1999). Endocrine and cytokine correlates of major depression and dysthymia with typical or atypical features. Molecular psychiatry, 4: 182-188

[29] Annane D, Sebille V, Charpentier C, Bollaert PE, Francois B, Korach JM, et al. (2002). Effect of treatment with low doses of hydrocortisone and fludrocortisone on mortality in patients with septic shock. Jama, 288: 862-871

[30] Jane JA, Rimel RW (1982). Prognosis in head injury. Clinical neurosurgery, 29: 346-352

[31] Rimel RW, Jane JA, Edlich RF (1979). An injury severity scale for comprehensive management of central nervous system trauma. Jacep, 8: 64-67

[32] Rimel RW, Giordani B, Barth JT, Jane JA (1982). Moderate head injury: completing the clinical spectrum of brain trauma. Neurosurgery, 11: 344-351

[33] Parikh S, Koch M, Narayan RK (2007). Traumatic brain injury. International anesthesiology clinics, 45: 119-135

[34] Kraus JF, Black MA, Hessol N, Ley P, Rokaw W, Sullivan C, et al. (1984). The incidence of acute brain injury and serious impairment in a defined population. American journal of epidemiology, 119: 186-201

[35] Stone JJ, Childs S, Smith LE, Battin M, Papadakos PJ,
Huang JH (2014). Hourly neurologic assessments for traumatic brain injury in the ICU. Neurological research, 36: 164-169

[36] Teasdale G, Jennett B (1974). Assessment of coma and impaired consciousness. A practical scale. Lancet, 2: 8184

[37] Kay T HD, Adams R, Berrol S, Cicerone K, Dahlberg C, et al. (1993). Definition of mild traumatic brain injury. J Head Trauma Rehabil, 8: 2

[38] Kosty JA, Stein SC (2013). Measuring outcome after severe TBI. Neurological research, 35: 277-284

[39] Jennett B, Bond M (1975). Assessment of outcome after severe brain damage. Lancet, 1: 480-484

[40] Association. AP (1994) Diagnostic and Statistical Manual of Mental Disorders. , American Psychiatric Association, Washington, DC

[41] Beck AT, Steer RA, Ball R, Ranieri W (1996). Comparison of Beck Depression Inventories -IA and -II in psychiatric outpatients. Journal of personality assessment, 67: 588-597

[42] Asnani MR, Fraser R, Lewis NA, Reid ME (2010). Depression and loneliness in Jamaicans with sickle cell disease. BMC psychiatry, 10: 40

[43] Beck AT SR, Brown GK. (1996) Manual for the BDI-II, Psychological Corporation, San Antonio, TX

[44] Fann JR, Hart T, Schomer KG (2009). Treatment for depression after traumatic brain injury: a systematic review. Journal of neurotrauma, 26: 2383-2402

[45] Delahanty DL, Gabert-Quillen C, Ostrowski SA, Nugent NR, Fischer B, Morris A, et al. (2013). The efficacy of initial hydrocortisone administration at preventing posttraumatic distress in adult trauma patients: a randomized trial. CNS spectrums, 18: 103-111

[46] Barth J, Munder T, Gerger H, Nuesch E, Trelle S, Znoj H, et al. (2013). Comparative efficacy of seven psychotherapeutic interventions for patients with depression: a network meta-analysis. PLoS medicine, 10: e1001454

[47] Fann JR, Bombardier CH, Temkin NR, Esselman P, Pelzer E, Keough M, et al. (2003). Incidence, severity, and phenomenology of depression and anxiety in patients with moderate to severe traumatic brain injury. Psychosomatics: 161

[48] Bourdon KH, Rae DS, Locke BZ, Narrow WE, Regier DA (1992). Estimating the prevalence of mental disorders in U.S. adults from the Epidemiologic Catchment Area Survey. Public health reports, 107: 663-668

[49] Fukami K, Yamagishi S, Okuda S (2014). Role of AGEsRAGE system in cardiovascular disease. Current pharmaceutical design, 20: 2395-2402

[50] Hutchison ER, Kawamoto EM, Taub DD, Lal A, Abdelmohsen K, Zhang Y, et al. (2013). Evidence for miR-181 involvement in neuroinflammatory responses of astrocytes. Glia, 61: 1018-1028

[51] Xue Q, Guo ZY, Li W, Wen WH, Meng YL, Jia LT, et al. (2011). Human activated CD4(+) T lymphocytes increase IL-2 expression by downregulating microRNA-181c. Molecular immunology, 48: 592-599 